\title{
Effect of particle-hole symmetry on the behavior of tracer and jump diffusion coefficients
}

\author{
J. J. Torrez Herrera, G. A. Ranzuglia, S. J. Manzi, ${ }^{*}$ and V. D. Pereyra \\ Departamento de Física, Instituto de Física Aplicada (INFAP)_CONICET, Universidad Nacional de San Luis, \\ Chacabuco 917, 5700 San Luis, Argentina
}

(Received 11 October 2012; revised manuscript received 28 February 2013; published 2 May 2013)

\begin{abstract}
This paper analyzes the effect of particle-hole symmetry on the behavior of the tracer diffusion coefficient as well as the jump diffusion coefficient. The coefficients are obtained by performing a random walk of individual atoms in a two-dimensional square lattice at monolayer, using the $n$-fold way Monte Carlo simulation. Different hopping mechanisms have been introduced to study the effect of particle-hole symmetry. For hopping kinetics where the initial-state interactions are involved, the diffusion coefficient at high coverage falls several orders of magnitude due to the effect of particle-hole symmetry. For hopping kinetics where the final-state interactions are present, the effect is the opposite. For those involving both initial- and final-state interactions, like the so-called interaction kinetics, the effect of particle-hole symmetry is also discussed. This effect seems to be critical for repulsive lateral interactions, for which the behavior of the diffusion coefficients is modified by introducing the particle-hole symmetry condition.
\end{abstract}

DOI: 10.1103/PhysRevE.87.052101

PACS number(s): 05.40.-a, 68.43.De, 68.43.Jk

\section{INTRODUCTION}

The theoretical description of the collective diffusion process is a complicated many-body problem and various approaches have been applied to it, ranging from analytic ones based on master, Fokker-Planck, or Kramers equations to numerical Monte Carlo simulations. An important background is provided in Refs. [1-7].

The kinetic lattice gas model (KLGM) is usually an adequate background in which to describe diffusion of individual or collective motion of atoms in one-, two-, or three-dimensional systems. Besides this approximation, the diffusion can be described, e.g., by using the FrenkelKontorova model [8].

In the framework of the KLGM, diffusion coefficients represent the most important nonequilibrium characteristics. The analysis of these coefficients can be based on the linearresponse theory [9-11] or gradient expansions of correlation functions $[12,13]$.

For an interacting two-dimensional lattice gas, important results were obtained considering the quasiequilibrium distribution [14] and the semiphenomenological approach [1]. One such result is the so-called Reed-Ehrlich (RE) factorization, where a simple expression for the chemical or collective diffusion coefficient was proposed as a product of the thermodynamic factor (which is proportional to the inverse of the compressibility of the adsorbed phase) and the kinetic or dynamic factor (the average transition rate).

Physically, diffusion jumps represent the simplest Arrhenius processes and can be described by the transition state theory (TST) $[15,16]$. This theory has been used to describe diffusion [16,17] and other processes [16,18-22]. In particular, it has been shown $[16,17]$ that following TST, one can derive the RE expression for the collective diffusion coefficient. Recently, many other approaches have been used [23,24].

A rather general approach has been proposed by Payne and Kreuzer (PK) [25] for one-dimensional (1D) and 2D diffusion

\footnotetext{
*sergiojmanzi@gmail.com
}

[26,27]. They have exactly corroborated the RE factorization [1] for any range of interactions and for any form of the hopping rates that satisfies the principle of detailed balance. As expected, this result agrees with that obtained in Ref. [17]. However, the principle of detailed balance is a necessary but not sufficient condition for physically acceptable behavior of the diffusion coefficient. Thus, additional constraints on the hopping rates must be introduced, as is demonstrate in Refs. [28,29].

The method introduced by PK allows the introduction of different hopping mechanisms. Moreover, one can also consider the introduction of particle-hole symmetry (PHS) in the transition rate.

Note that a lattice gas possesses PHS if the Hamiltonian, a function of the site occupation numbers $n_{i}=0,1$, satisfies the relation $H \rightarrow H+A \sum_{i} n_{i}+B$ (with $A, B$ constants) under the transformation $n_{i} \rightarrow\left(1-n_{i}\right)$, while, for instance, real (continuous) fluids lack PHS ipso facto since a hole is not defined [30]. A lattice gas (at monolayer) where the PHS is fulfilled presents a symmetrical phase diagram (critical temperature versus surface coverage) around half-coverage [31].

For the hopping process, the PHS is fulfilled when the transition rate for a given configuration is the same as the transition rate for the same configuration, when particles are changed by holes. This was first discussed by PK for onedimensional systems in Ref. [25]. However, the generalization to two dimensions is not straightforward and must be carefully analyzed.

The aim of this work is to analyze the effect of PHS on the diffusion coefficients. Using the PK method, the transition rates are written as an expansion of the occupation configurations of all neighboring sites. Free coefficients arise from this expansion. Some of them are determined from the principle of detailed balance and the PHS condition. Therefore, an ad hoc dynamic scheme to get the rest of them is necessary to be introduced.

Three different hopping mechanisms are considered. First, we consider the initial state kinetics, where the hopping 
probability of a particle is determined by the interaction with its neighbors in the initial state. This is the usual pattern in the literature. The second mechanism is the final state kinetics, where the hopping probability of a particle is determined by the interaction with their neighbors in the final state. Finally, we consider the interaction kinetics, where the hopping probability of a particle is determined by the interaction with its neighbors in the initial and final state [25].

As is demonstrated in the present work, the influence of the PHS in the three above-described schemes plays an important role. All the results are obtained by means of $n$-fold way Monte Carlo simulations in a two-dimensional square lattice. Repulsive interaction between nearest-neighbor particles to analyze the diffusion coefficients in the vicinity of the orderdisorder phase transition is considered.

The outline of the paper is as follows. In Sec. II, the kinetic lattice gas model is described, introducing the master equation formalism. Then, the principle of detailed balance and PHS are used to determine the relations between the different parameters involved. In Sec. III, the definitions of the collective, tracer, and jump diffusion coefficients are introduced. In Sec. IV, the results are analyzed. Finally, in Sec. V, a summary and the conclusions are given.

\section{THE KINETIC LATTICE GAS MODEL}

Let us consider a system composed of $M$ sites in a twodimensional square lattice of linear size $L$. To describe the state of the system, one introduces microscopic occupation numbers $n_{i, j}=1$ or 0 , depending on whether the site $(i, j)$ is occupied by an adsorbed particle or not, respectively. There are $2^{M}$ microstates given by sequences of zeros and ones. To introduce the dynamics of the system, one writes down a model Hamiltonian:

$$
H=E_{s} \sum_{i, j} n_{i, j}+\frac{V}{2} \sum_{i, j ; i^{\prime}, j^{\prime}} n_{i, j} n_{i^{\prime}, j^{\prime}}+\cdots,
$$

where $\left(i^{\prime}, j^{\prime}\right)$ are the indexes corresponding to the nearestneighbor sites of site $(i, j)$. Since the lattice gas Hamiltonian should give the same Helmholtz free energy as a microscopic Hamiltonian (for noninteracting particles), one can show that the proper identification of $E_{S}$ is the free energy per particle [32],

$$
E_{s}=-V_{0}-k_{B} T \ln \left(q_{3} q_{\text {int }}\right),
$$

where $V_{0}$ is the depth of the surface potential. The centerof-mass vibrations of the adsorbed molecule in this potential well are represented by the partition functions $q_{3}=q_{z} q_{x y}$, with normal $(z)$ and in-plane $(x, y)$ components; $q_{\text {int }}=q_{\text {vib }} q_{\text {rot }}$ is the partition function for the internal degrees of freedom. $V$ is the interaction between nearest-neighbor particles. Longer-range interactions, like next-nearest-neighbor and trio interactions, can be included [25-27].

Let us introduce a function $P(\mathbf{n}, t)$, which gives the probability that a given microstate $\mathbf{n}=\left\{n_{1,1}, \ldots, n_{i, j}, \ldots, n_{L, L}\right\}$ of the lattice gas is realized at time $t$, and the function $W\left(\mathbf{n} ; \mathbf{n}^{\prime}\right)$, which gives the transition probability per unit time and is obtained as the probability to go from state $\mathbf{n}^{\prime}$ to state $\mathbf{n}$ per unit time. Treating hopping as a Markov process, $P(\mathbf{n}, t)$ must satisfy a master equation,

$$
\frac{d P(\mathbf{n} ; t)}{d t}=\sum_{n^{\prime}}\left[W\left(\mathbf{n} ; \mathbf{n}^{\prime}\right) P\left(\mathbf{n}^{\prime} ; t\right)-W\left(\mathbf{n}^{\prime} ; \mathbf{n}\right) P(\mathbf{n} ; t)\right] .
$$

To ensure the approach to equilibrium in an isolated system, each term in the sum must satisfy the principle of detailed balance:

$$
W\left(\mathbf{n}^{\prime} ; \mathbf{n}\right) P_{\mathrm{eq}}(\mathbf{n})=W\left(\mathbf{n} ; \mathbf{n}^{\prime}\right) P_{\mathrm{eq}}\left(\mathbf{n}^{\prime}\right),
$$

where

$$
P_{\mathrm{eq}}(\mathbf{n})=\frac{e^{-[H(\mathbf{n})-\mu N(\mathbf{n})] / k_{B} T}}{\Xi} .
$$

Here, $k_{B}, T$, and $\mu$ are the Boltzmann constant, the temperature, and the chemical potential, respectively. $P_{\text {eq }}$ denotes the equilibrium probability and $\Xi$ is the grand-canonical partition function given by

$$
\Xi=\sum_{n} e^{-[H(\mathbf{n})-\mu N(\mathbf{n})] / k_{B} T} .
$$

In the absence of adsorption and desorption, the number of adparticles, $N(\mathbf{n})$, is fixed and the form of $W\left(\mathbf{n}^{\prime} ; \mathbf{n}\right)$ depends on the hopping scheme. We restrict our description of diffusion to that resulting from the hopping of single particles from an occupied site to a neighboring unoccupied site. Then we can write this transition probability as [25-27,33]

$$
\begin{aligned}
W_{\mathrm{diff}}\left(\mathbf{n}^{\prime} ; \mathbf{n}\right)= & \sum_{i, j ; i^{\prime}, j^{\prime}}\left[W_{i, j}^{>}(\mathbf{n})+W_{i^{\prime}, j^{\prime}}^{<}(\mathbf{n})\right] \\
& \times \delta_{n_{i, j}^{\prime}}^{1-n_{i, j}} \delta_{n_{i^{\prime}, j^{\prime}}^{\prime}}^{1-n_{i^{\prime}, j^{\prime}}} \prod_{\substack{l, k \neq i, j \\
l, k \neq i^{\prime}, j^{\prime}}} \delta_{n_{l, k}^{\prime}}^{n_{l, k}}
\end{aligned}
$$

Then, the transition rate $W_{i, j}^{>}$from site $(i, j)$ to site $(i, j+1)$ can be written in compact form as

$$
W_{i, j}^{>}(\mathbf{n})=J_{0} n_{i, j}\left(1-n_{i, j+1}\right)\left[1+\mathbf{A} \mathbf{N}+\mathbf{B M}+\mathbf{S}^{\mathbf{T}} \mathbf{C}^{\mathbf{T}} \mathbf{R}\right],
$$

where $J_{0}$ is the elementary hopping rate for a single particle at zero coverage (usually it has Arrhenius form). The rate $W_{i, j}^{<}$gives the reverse current and it can be obtained with the interchanges $j \rightleftarrows j+1$ and $j-1 \rightleftarrows j+2$. In the preceding equation, $\mathbf{A}$ is a $1 \times 3$ matrix,

$$
\mathbf{A}=\left(\begin{array}{lll}
A_{1} & A_{2} & A_{3}
\end{array}\right),
$$

with components $A_{s}$ which take into account the effect of the interactions of the hopping particle with its $s$ neighbors in the initial state. Likewise, matrix $\mathbf{B}$ is a $1 \times 3$ matrix,

$$
\mathbf{B}=\left(\begin{array}{lll}
B_{1} & B_{2} & B_{3}
\end{array}\right),
$$

whose components $B_{s}$ take into account the effect of the interactions of the hopping particle with its $s$ neighbors in the final state.

On the other hand, $\mathbf{N}$ and $\mathbf{M}$ are $3 \times 1$ matrices which represent the occupation state of the nearest-neighbor sites 
involved in the process,

$$
\mathbf{N}=\left(\begin{array}{c}
n_{i, j-1}+n_{i-1, j}+n_{i+1, j} \\
n_{i, j-1}\left(n_{i-1, j}+n_{i+1, j}\right)+n_{i-1, j} n_{i+1, j} \\
n_{i, j-1} n_{i-1, j} n_{i+1, j}
\end{array}\right)
$$

and

$$
\mathbf{M}=\left(\begin{array}{c}
n_{i-1, j+1}+n_{i+1, j+1}+n_{i, j+2} \\
n_{i, j+2}\left(n_{i-1, j+1}+n_{i+1, j+1}\right)+n_{i-1, j+1} n_{i+1, j+1} \\
n_{i, j+2} n_{i-1, j+1} n_{i+1, j+1}
\end{array}\right) .
$$

To consider the PHS condition, is necessary to introduce the following $3 \times 1$ matrices:

$$
\mathbf{S}=\left(\begin{array}{c}
n_{i+2, j}\left(1-n_{i+1, j+1}\right)\left(1-n_{i+1, j-1}\right)+\left(1-n_{i+2, j}\right) n_{i+1, j+1}\left(1-n_{i+1, j-1}\right)+\left(1-n_{i+2, j}\right)\left(1-n_{i+1, j+1}\right) n_{i+1, j-1} \\
n_{i+2, j}\left[n_{i+1, j+1}\left(1-n_{i+1, j-1}\right)+n_{i+1, j-1}\left(1-n_{i+1, j+1}\right)\right]+n_{i+1, j+1} n_{i+1, j-1}\left(1-n_{i+2, j}\right) \\
n_{i+2, j} n_{i+1, j+1} n_{i+1, j-1}
\end{array}\right)
$$

and

$$
\mathbf{R}=\left(\begin{array}{c}
n_{i, j+2}\left(1-n_{i-1, j+1}\right)\left(1-n_{i+1, j+1}\right)+n_{i-1, j+1}\left(1-n_{i, j+2}\right)\left(1-n_{i+1, j+1}\right)+n_{i+1, j+1}\left(1-n_{i-1, j+1}\right)\left(1-n_{i, j+2}\right) \\
n_{i, j+2}\left[n_{i-1, j+1}\left(1-n_{i+1, j+1}\right)+n_{i+1, j+1}\left(1-n_{i-1, j+1}\right)\right]+n_{i-1, j+1} n_{i+1, j+1}\left(1-n_{i, j+2}\right) \\
n_{i, j+2} n_{i-1, j+1} n_{i+1, j+1}
\end{array}\right) .
$$

Finally, $\mathbf{C}$ is a $3 \times 3$ matrix:

$$
\mathbf{C}=\left(\begin{array}{lll}
C_{11} & C_{12} & C_{13} \\
C_{21} & C_{22} & C_{23} \\
C_{31} & C_{32} & C_{33}
\end{array}\right),
$$

where each term $C_{k l}$ represents the interaction of a system composed of one particle occupying the site $(i, j)$ surrounded by $k$ occupied nearest-neighbor sites, and its nearest-neighbor empty site $\left(i^{\prime}, j^{\prime}\right)$ surrounded by $l$ occupied nearest-neighbor sites.

To clarify how Eq. (8) works, in Fig. 1 an example is shown. In the rate transition, the term $3 A_{1}$ is due to the three occupied nearest-neighbor sites of the hopping particle; the term $3 A_{2}$ is

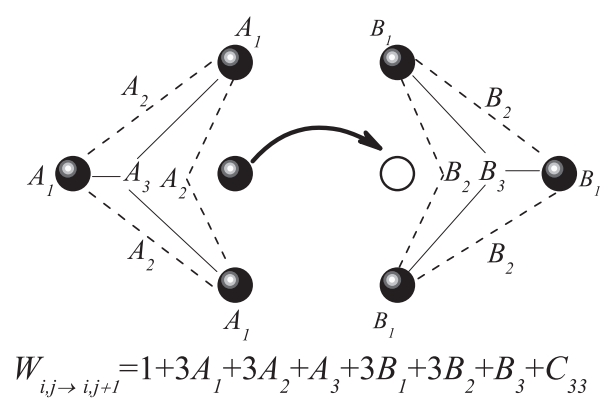

FIG. 1. An example of the hopping process and the transition rate. Dashed lines denote the pair contribution, and solid lines denote the trio contribution. the contribution of the pairs of occupied nearest-neighbor sites of the hopping particle, and these are indicated by dashed lines in the figure; and the term $A_{3}$ is due to the triplet of occupied nearest-neighbor sites of the hopping particle (solid lines). Similarly, considering the final state of the hopping particle and the interactions with the occupied nearest neighbors, one can obtain the terms corresponding to the coefficients $B_{s}$. Finally, the term $C_{33}$ is because both the initial and final state of the hopping particle have three occupied nearest neighbors.

The principle of detail balance imposes the following restrictions on the coefficients of rate transitions:

$$
\begin{gathered}
\left(1+B_{1}\right) u=\left(1+A_{1}\right), \\
\left(1+2 B_{1}+B_{2}\right) u^{2}=\left(1+2 A_{1}+A_{2}\right), \\
\left(1+3 B_{1}+3 B_{2}+B_{3}\right) u^{3}=\left(1+3 A_{1}+3 A_{2}+A_{3}\right), \\
\left(1+A_{1}+2 B_{1}+B_{2}+C_{12}\right) u \\
=\left(1+2 A_{1}+A_{2}+B_{1}+C_{21}\right), \\
\left(1+A_{1}+3 B_{1}+3 B_{2}+B_{3}+C_{13}\right) u^{2} \\
=\left(1+3 A_{1}+3 A_{2}+A_{3}+B_{1}+C_{31}\right), \\
\left(1+2 A_{1}+A_{2}+3 B_{1}+3 B_{2}+B_{3}+C_{23}\right) u \\
=\left(1+3 A_{1}+3 A_{2}+A_{3}+2 B_{1}+B_{2}+C_{32}\right),
\end{gathered}
$$

where $u=\exp \left(V / k_{B} T\right)$. 
If PHS is to be preserved in the hopping process, the following relations have to be fulfilled too:

$$
\begin{gathered}
3 A_{1}+3 A_{2}+A_{3}+3 B_{1}+3 B_{2}+B_{3}+C_{33}=0, \\
2 A_{1}+3 A_{2}+A_{3}+2 B_{1}+B_{2}+C_{32}=0, \\
A_{1}+2 A_{2}+A_{3}+B_{1}+C_{31}=0, \\
2 A_{1}+A_{2}+2 B_{1}+3 B_{2}+B_{3}+C_{23}=0, \\
A_{1}+B_{1}+2 B_{2}+B_{3}+C_{13}=0, \\
A_{1}+A_{2}+B_{1}+B_{2}+C_{22}-C_{11}=0 .
\end{gathered}
$$

Equations (16)-(27) are obtained considering two nearestneighbor sites, one occupied by the hopping particle and the other by a vacancy; they are surrounded by its six nearest neighbors. The rest of the lattice is frozen during the hopping event. For example, Eq. (16) arises from considering the transition between two configurations containing two particles, one where the hopping particle has one occupied nearest neighbor, and the other where the hopping particle is surrounded by vacancies. There are six pairs of configurations which give the same balance equation. Similarly, Eqs. (17)-(21) are obtained. Note that the number of occupied nearest neighbors for the hopping particle must be different from the number of occupied nearest neighbors of the vacancy; otherwise, the balance equations between configurations cancel each other out.

Let us explain Eqs. (22)-(27). As we mentioned in the Introduction, the particle-hole symmetry is fulfilled when the transition rate for a given configuration is the same as the transition rate for the same configuration, when particles are changed by holes (vacancies). For example, Eq. (22) arises from the PHS applied to the configuration showed in Fig. 1. In fact, the transition rate for the particle is written in the figure, while that corresponding to the vacancy is $W=1$. From equating both transition rates, Eq. (22) can be obtained. In the Appendix, the configurations leading to the rest of the equations (23)-(27) corresponding to PHS conditions are shown. Note that the rate transitions between those configurations in which the number of empty (occupied) sites surrounding the hopping particle is the same as the number of occupied (empty) sites surrounding the hopping vacancy cancel each other out.

\section{THE TRACER AND JUMP DIFFUSION COEFFICIENTS}

The collective diffusion of atoms can also be described by the Kubo-Green theory. In the framework of this theory, a first approach to calculate the collective diffusion coefficient is as a product of the jump diffusion coefficient and the so-called thermodynamic factor. The jump and tracer diffusion coefficients of surface species involve jumps of adsorbed interacting particles from one binding site to another. The jump diffusion coefficient $D_{J}(\theta)$, accounting for the effective hopping kinetics in an interacting system, gives a measurement of the movement of the center of mass of the adsorbed particles, while the tracer diffusion coefficient, $D_{T}(\theta)$, is visualized as a measurement of the random walk of individual particles.
Both coefficients are obtained by performing a random walk of individual atoms on the substrate. This random walk characterizes the diffusion of surface particles and is strongly determined by the hopping schemes considered. For this reason, Monte Carlo simulation seems to be the most natural tool to analyze the problem.

The tracer diffusion coefficient, $D_{T}$, is determined from measurements of the mean-square displacements (MSDs) of the $N$ tagged adatoms, which for a $d$-dimensional system agree with $[1,2,34]$

$$
D_{T}=\lim _{t \rightarrow \infty}\left[\frac{1}{2 d N t}\left\langle\left(\sum_{i=1}^{N}\left[\mathbf{r}_{i}(t)-\mathbf{r}_{i}(0)\right]^{2}\right)\right\rangle\right] .
$$

In the framework of the Kubo-Green theory [1,2], the collective diffusion coefficient can be written, in a first approximation, as

$$
D_{C}(\theta, T)=\left(\frac{\partial \mu / k_{B} T}{\partial \ln \theta}\right) D_{J}=\left[\frac{\langle\delta N\rangle}{\langle N\rangle}\right]^{-1} D_{J},
$$

where $\theta=\langle N\rangle / L^{d}$ is the coverage and $\langle\delta N\rangle$ is the meansquare fluctuations in an area $A$ containing $\langle N\rangle$ particles. Precisely, $\left[\frac{\langle\delta N\rangle}{\langle N\rangle}\right]^{-1}=\chi^{-1}$ is the so-called thermodynamic factor. Here $\chi^{-1}$ is the inverse of the surface susceptibility. The jump diffusion coefficient, $D_{J}$, is related to the MSD of the center of mass of the system by

$$
D_{J}=\lim _{t \rightarrow \infty}\left[\frac{1}{2 d N t}\left\langle\left(\sum_{i=1}^{N}\left|\mathbf{r}_{i}(t)-\mathbf{r}_{i}(0)\right|\right)^{2}\right\rangle\right] .
$$

Usually, to obtain $D_{T}$ and $D_{J}$, the MSD of individual particles and the MSD of the center of mass of the system as a function of time are plotted in log-log form. Then, taking the slope of each curve for very large times, provided that normal diffusion is present, from the intercept, one gets the value of $D_{J}$ and $D_{T}[35,36]$.

Normal or Gaussian diffusion in a one-dimensional system means that $D_{J} \propto t^{\omega}$ (with $\omega=1$ ), while it is demonstrated [37] that $D_{T} \propto t^{1 / 2}$. However, for a two-dimensional system, the diffusion exponents for both coefficients, $D_{J}$ and $D_{T}$, are equal to $1, \omega=1$ (for more information, see Ref. [4]).

To perform the Monte Carlo simulation describing the diffusion process, an $n$-fold scheme was used [35]. Each particle has four transition rates, which are evaluated through Eq. (8). This algorithm has been shown to be very efficient below critical temperature. In the next section, the results for two-dimensional systems are presented.

\section{RESULTS}

The coverage and temperature dependence for both the $D_{T}$ and $D_{J}$ coefficients for a two-dimensional system are discussed. The system is represented by a square lattice with periodic boundary conditions. Only repulsive lateral interactions between nearest neighbors are considered.

As is demonstrated in Ref. [22], the present dynamics belongs to the so-called soft dynamics, that is, the transition rates depend on the parameters $A_{i}, B_{i}$, and $C_{i}$, which are only functions of the lateral interaction $V$ and temperature $T$. 
They do not depend on $E_{s}$ and $V_{0}$, therefore the values of such parameters do not influence the behavior of the diffusion coefficients.

It is well known that for nearest-neighbor repulsive lateral interactions, the adsorbate undergoes a phase transition from disorder to a $c(2 \times 2)$ ordered phase for a certain range of coverages $(0.36<\theta<0.64$, at $T=0)$ [31,38-40]. Below the critical temperature, $T<T_{c}$ (for square lattices, $T_{c}$ is given by $\left.\frac{k_{B} T_{c}}{V}=0.56729 \ldots\right)$, the thermodynamical factor has a sharp peak at $\theta=0.5$ and two minima at coverages corresponding to the entrance in the ordered phase. The jump and tracer diffusion coefficients are also influenced by the order and they change their behavior at $T=T_{c}$. Next, the behavior of the tracer and jump diffusion coefficients for different hopping kinetics will be analyzed, particularly when initial- and finalstate interactions are involved.

First, the hopping kinetics involving initial-state interactions is considered. Thus, $B_{i}=0$ must be fulfilled. After solving Eqs. (16)-(18), we obtain the expressions for the $A_{i}$ coefficients:

$$
\begin{gathered}
A_{1}=e^{-\beta V}-1, \\
A_{2}=e^{-2 \beta V}-2 e^{-\beta V}+1, \\
A_{3}=e^{-3 \beta V}+3 e^{-\beta V}-3 e^{-2 \beta V}-1 .
\end{gathered}
$$

Consider first that all $C_{i j}=0$. Here, the PHS is not preserved, which is the situation most commonly used in the literature.

In Figs. 2(a) and 2(b), $D_{T}^{*}(\theta, \tau)$ and $D_{J}^{*}(\theta, \tau)$ versus coverage and reduced temperature, $\tau=T / T_{c}$, are shown. The behavior of both coefficients is very similar despite their different nature. At low coverage, the coefficients are slightly greater than the Langmuir case (noninteracting particles), and then they fall to very low values near half-coverage. In Ref. [11,34], it was shown that this is due to the presence of nearest-neighbor repulsive interactions, which causes the $c(2 \times 2)$ order of the system at temperatures around $T_{c}$. Both coefficients decrease as the temperature decreases below $T_{c}$. This result can be explained by the long-range $c(2 \times 2)$ order at half-coverage. It is quite apparent that this ordering is still rather imperfect at $T_{c}$ but substantially improves at lower temperatures. However, the motion of adatoms requires locally breaking the order, and this becomes increasingly difficult if the ordering is improving for temperatures well below $T_{c}$. Without breaking the order, the particles can perform flip-flop-like motions between adjacent lattice sites, but they are not able to diffuse around. Thus, the motion of particles in the $c(2 \times 2)$ ordered state is highly correlated. As coverage increases, additional particles occupy sites with high hopping probability. This is because this probability depends on the initial-state interaction. For this reason, diffusion increases with coverage.

Next, the results for the final-state hopping kinetics are presented. In this case, the jump is determined by the interactions in the final state, therefore $A_{i}=0$ must be fulfilled. The expressions of the $B_{i}$ coefficients are

$$
B_{1}=e^{\beta V}-1,
$$
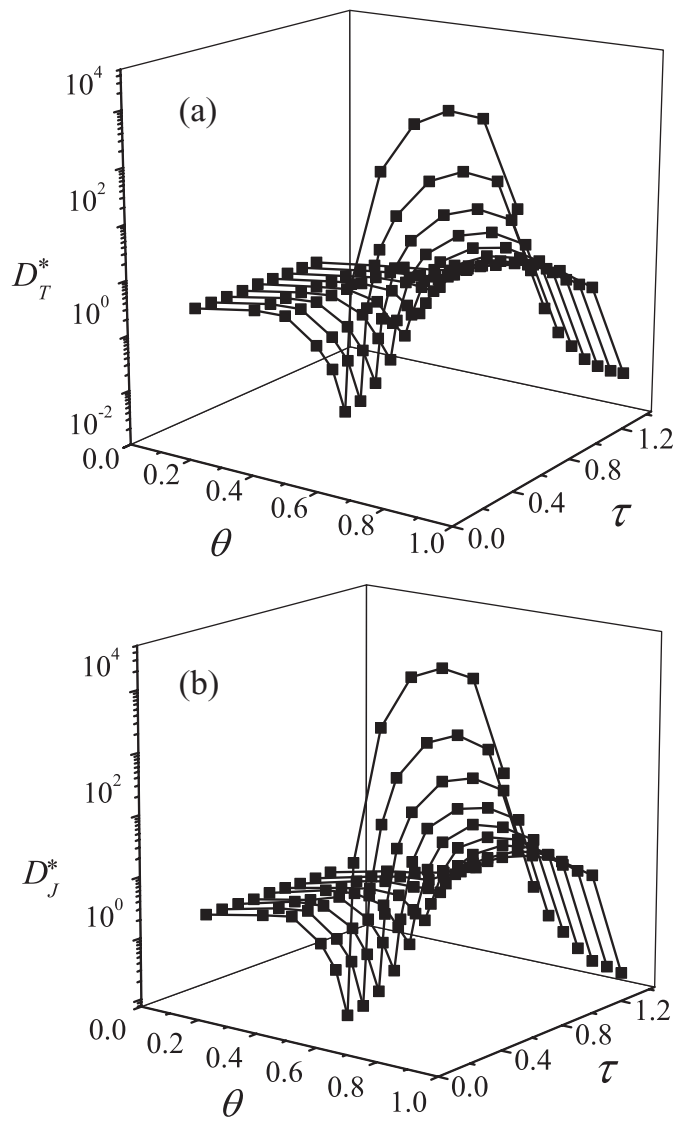

FIG. 2. (a) Normalized tracer diffusion coefficient and (b) normalized jump diffusion coefficient vs $\theta$ and $\tau$ for the hopping kinetics with initial-state interactions. The lower temperature corresponds to $\tau=0.3$ and the temperature step is $\Delta \tau=0.1$.

$$
\begin{gathered}
B_{2}=e^{2 \beta V}+1-2 e^{\beta V}, \\
B_{3}=e^{3 \beta V}-1+3 e^{\beta V}-3 e^{2 \beta V} .
\end{gathered}
$$

Here, let us consider the case $C_{i j}=0$. With this, the PHS is not preserved. In Figs. 3(a) and 3(b), the tracer and jump diffusion coefficients, for the hopping kinetics with final-state interactions, are plotted. In this case, the hopping probability depends on the final configuration. Below the critical temperature, the ordered phase is formed, the hopping probability falls significatively, and the diffusion coefficients decrease to very low values. The minimum occurs at half-coverage, where a perfect $c(2 \times 2)$ phase is formed. As coverage increases, the coefficients are slightly higher, and then they fall to near zero for high coverage. This effect is more marked at low temperatures.

Now, we add the effect of the PHS. To preserve the PHS, the corresponding coefficients $C_{i j}$ must be obtained from Eqs. (19)-(27). For initial-state hopping kinetics, they are given by

$$
\begin{gathered}
C_{11}=C_{12}=C_{13}=-A_{1}, \\
C_{21}=-A_{1} e^{\beta V}, \\
C_{22}=C_{23}=-A_{1}\left(1+e^{\beta V}\right),
\end{gathered}
$$



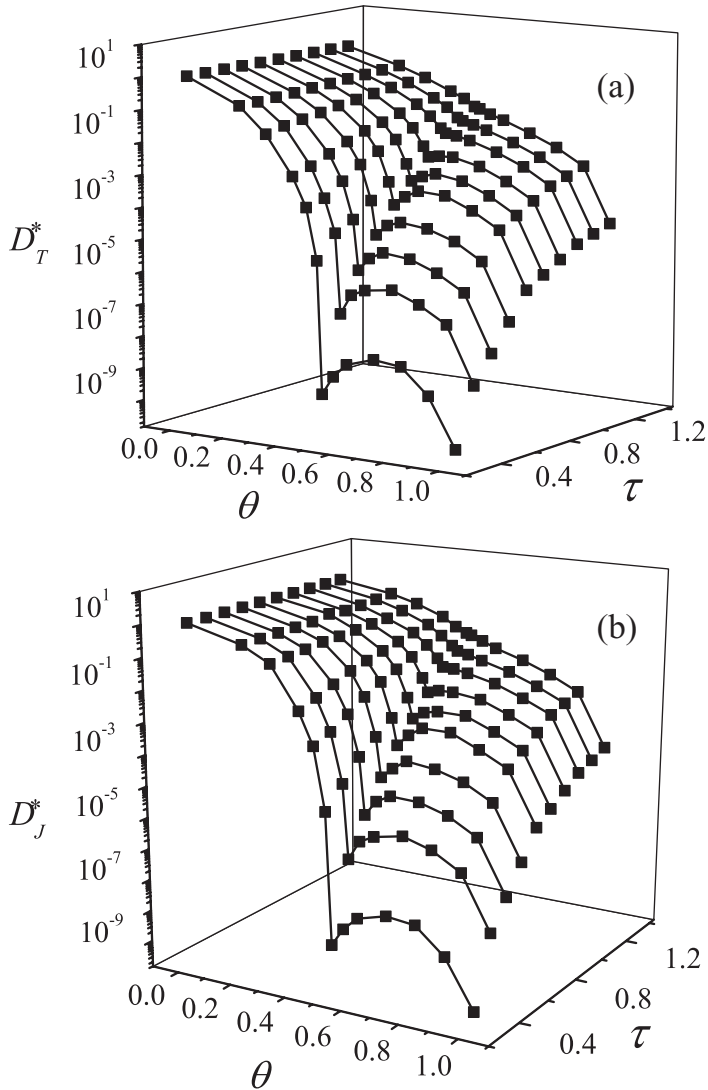

FIG. 3. (a) Normalized tracer diffusion coefficient and (b) normalized jump diffusion coefficient vs $\theta$ and $\tau$ for the hopping kinetics with final-state interactions. The lower temperature corresponds to $\tau=0.3$ and the temperature step is $\Delta \tau=0.1$.

$$
\begin{gathered}
C_{31}=-A_{1} e^{2 \beta V}, \\
C_{32}=-A_{1} e^{\beta V}\left(1+e^{\beta V}\right), \\
C_{33}=1-e^{3 \beta V},
\end{gathered}
$$

while for final-state hopping kinetics, the $C_{i j}$ coefficients are

$$
\begin{gathered}
C_{11}=C_{12}=C_{31}=-B_{1}, \\
C_{21}=-B_{1} e^{\beta V}, \\
C_{22}=C_{32}=-B_{1}\left(1+e^{-\beta V}\right), \\
C_{13}=-B_{1} e^{-2 \beta V}, \\
C_{23}=-B_{1} e^{-\beta V}\left(1+e^{-\beta V}\right), \\
C_{33}=1-e^{-3 \beta V} .
\end{gathered}
$$

Note that Eqs. (16)-(27) form a $12 \times 12$ indeterminate linear system, where two coefficients, $C_{21}$ and $C_{22}$, must be introduced in an ad hoc way [see Eqs. (19) and (27)] [41]. In Fig. 4, the normalized jump diffusion coefficients corresponding to $C_{i j}=0$ (open circles), and different choices of $C_{i j} \neq 0$ (filled symbols), for initial- and final-state hopping kinetics, are compared. As is observed, the PHS introduces

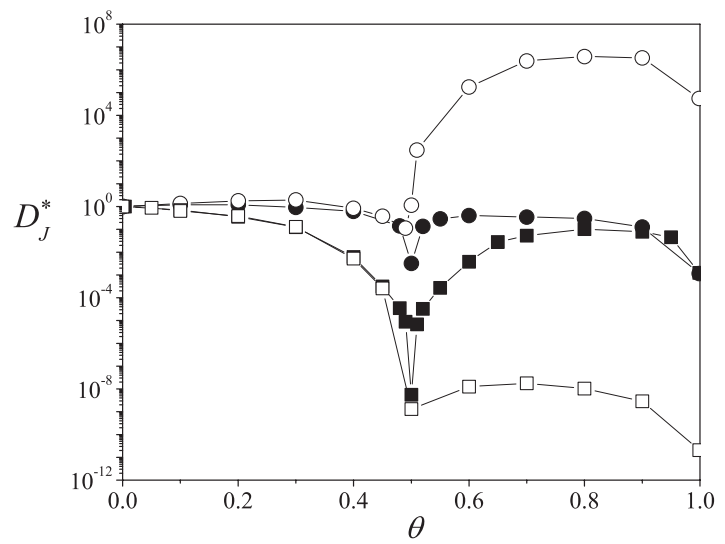

FIG. 4. Comparison between the normalized jump diffusion coefficients calculated by using $C_{i j}=0$ (open symbols) and with $C_{i j} \neq 0$ (filled symbols) for $\tau=0.3$. The circles correspond to the initial-state kinetics, while the squares correspond to the final-state kinetics.

a strong effect on diffusion coefficients at high coverages. Indeed, the variation of the coefficients covers more than seven orders of magnitude at $\theta \approx 0.75$, depending on whether or not it meets the PHS. At low coverages, the PHS does not introduce significant changes in the diffusion coefficients.
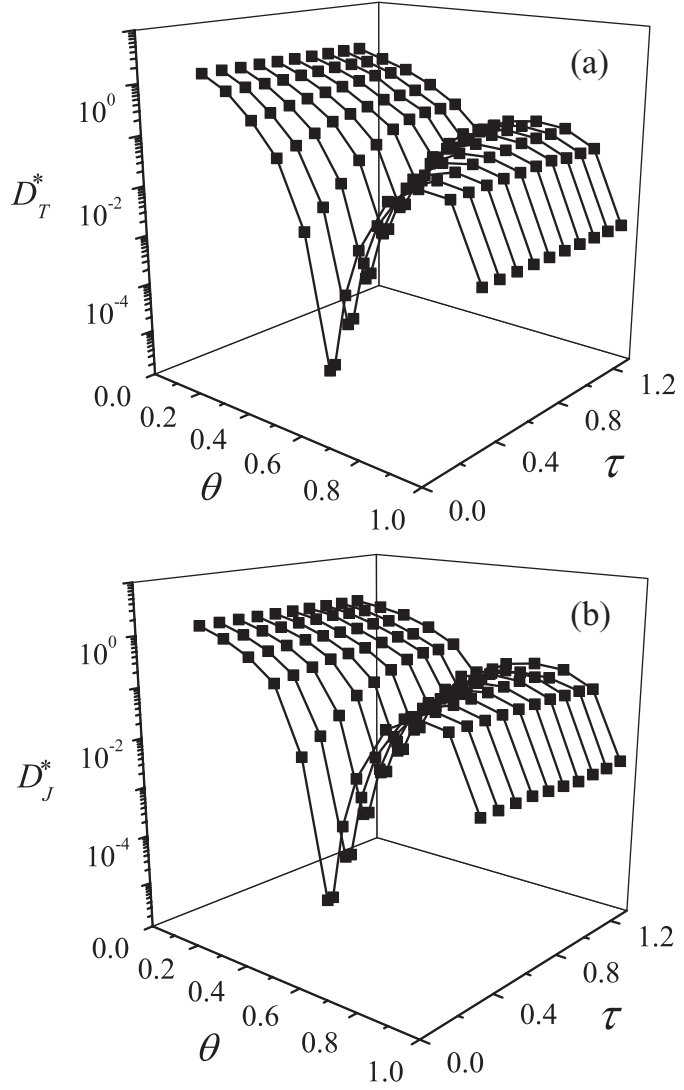

FIG. 5. (a) Normalized tracer diffusion coefficient and (b) normalized jump diffusion coefficient vs $\theta$ and $\tau$ for the interaction kinetics with $\gamma=1$ and $C_{i j} \neq 0$. The lower temperature corresponds to $\tau=0.3$ and the temperature step is $\Delta \tau=0.1$. 


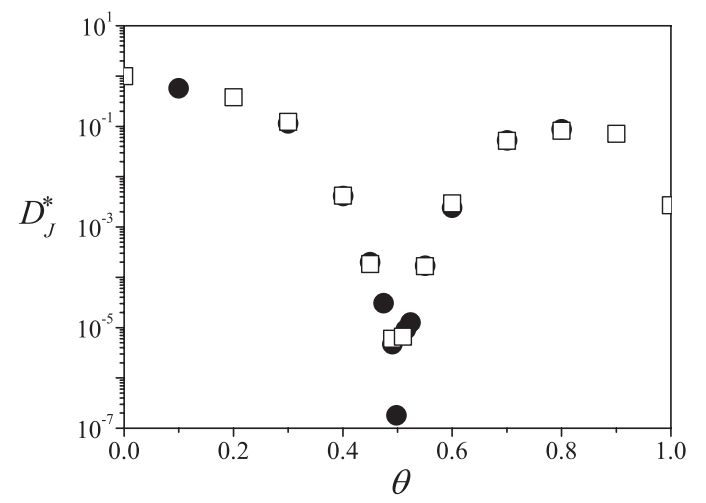

FIG. 6. Comparison between normalized jump diffusion coefficient calculated by using the interaction kinetics (open squares) with $\gamma=1$ and $C_{i j} \neq 0$ and that obtained by using a simple METROPOLIS scheme (filled circles).

Next, the so-called interaction kinetics introduced in Ref. [25] is discussed. The corresponding coefficients are related to each other by

$$
A_{i}=-\gamma B_{i}
$$

Then, after using the principle of detailed balance [Eqs. (16)(18)], the expressions for the $B_{i}$ coefficients are

$$
\begin{gathered}
B_{1}=\frac{1-e^{\beta V}}{\gamma+e^{\beta V}}, \\
B_{2}=\frac{1-e^{2 \beta V}}{\gamma+e^{2 \beta V}}-2 B_{1}, \\
B_{3}=\frac{1-e^{3 \beta V}}{\gamma+e^{3 \beta V}}-3 B_{1}-3 B_{2} .
\end{gathered}
$$

According to the same criteria as those used in the onedimensional case, the phase diagram showing the values of $\gamma$ and $V$, where the diffusion coefficients are positive or negative, can be obtained; see Ref. [29]. Taking $\gamma=1$, the diffusion coefficients are always positive.

To analyze the effect of the PHS condition, the case in which the coefficients $C_{i j} \neq 0$ is considered. A possible choice of the

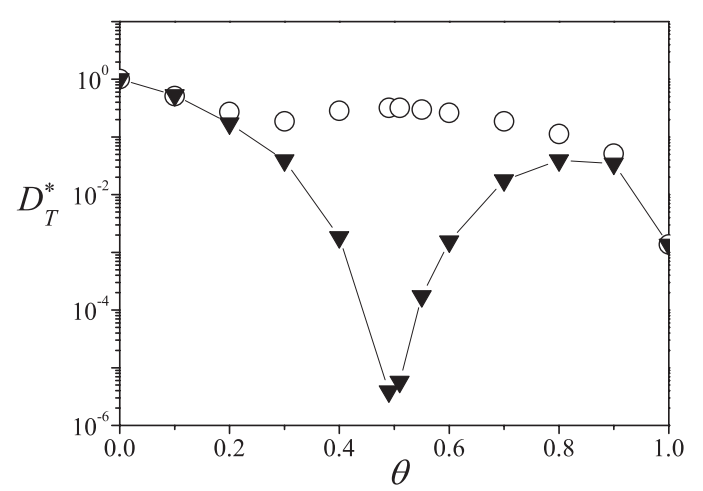

FIG. 7. Comparison between the normalized tracer diffusion for the interaction kinetics with $\gamma=1, \tau=0.3$, and different PHS conditions ( $C_{i j} \neq 0$, solid symbols; $C_{i j}=0$, open symbols).
TABLE I. Comparison between the transition rates for the interaction kinetics with $\gamma=1$ and a different PHS condition.

\begin{tabular}{lcc}
\hline \hline$[i][f]$ & $W_{\text {diff }}\left(C_{i j}=0\right)$ & $W_{\text {diff }}\left(C_{i j} \neq 0\right)$ \\
\hline$[1][2]$ & 0.9051 & 0.1005 \\
{$[1][3]$} & 0.8998 & 0.0056 \\
{$[2][1]$} & 1.0949 & 1.8995 \\
{$[2][3]$} & 0.9947 & 0.1004 \\
{$[3][1]$} & 1.0018 & 1.9944 \\
{$[3][2]$} & 1.0053 & 1.8995 \\
\hline
\end{tabular}

coefficients that satisfies all of Eqs. (19)-(27) may be

$$
\begin{gathered}
C_{11}=B_{1}(\gamma-1), \\
C_{12}=B_{1}(\gamma-1)-B_{2}, \\
C_{13}=B_{1}(\gamma-1)-2 B_{2}-B_{3}, \\
C_{21}=B_{1}(\gamma-1)+\gamma B_{2}, \\
C_{22}=2 B_{1}(\gamma-1)+B_{2}(\gamma-1), \\
C_{23}=2 B_{1}(\gamma-1)+B_{2}(\gamma-3)-B_{3}, \\
C_{31}=B_{1}(\gamma-1)+2 \gamma B_{2}+\gamma B_{3}, \\
C_{32}=2 B_{1}(\gamma-1)+B_{2}(3 \gamma-1)+\gamma B_{3}, \\
C_{33}=3 B_{1}(\gamma-1)+3 B_{2}(\gamma-1)+B_{3}(\gamma-1) .
\end{gathered}
$$

Again, because the number of equations is not enough to get all coefficients independently, some of them must be defined in an ad hoc way, specifically $C_{22}$ and $C_{12}$ [41].

In Figs. 5(a) and 5(b), the normalized tracer and jump diffusion coefficients, for $\gamma=1$ and $C_{i j}$ given by Eqs. (53)-(61), are plotted. The reduced temperature goes from $\tau=0.3$ to 1.2 in steps of $\Delta \tau=0.1$. Since the hopping mechanism takes into account the initial- and final-state

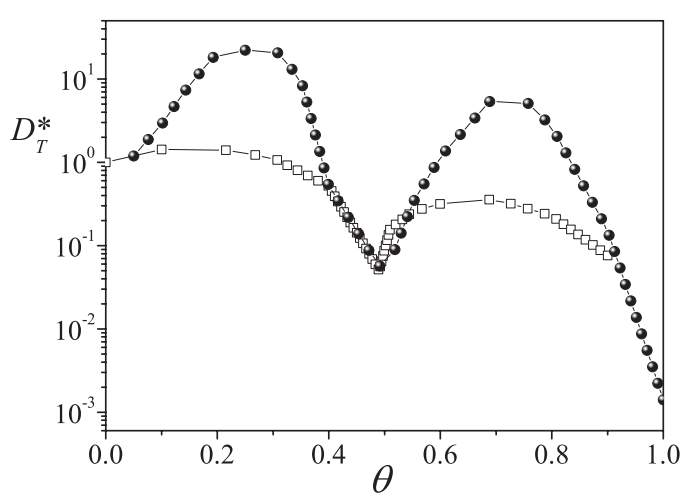

FIG. 8. Comparison between the normalized tracer diffusion coefficient calculated using the initial-state kinetics: $C_{11}=0$, open squares; $C_{22}=0$, solid circles. 

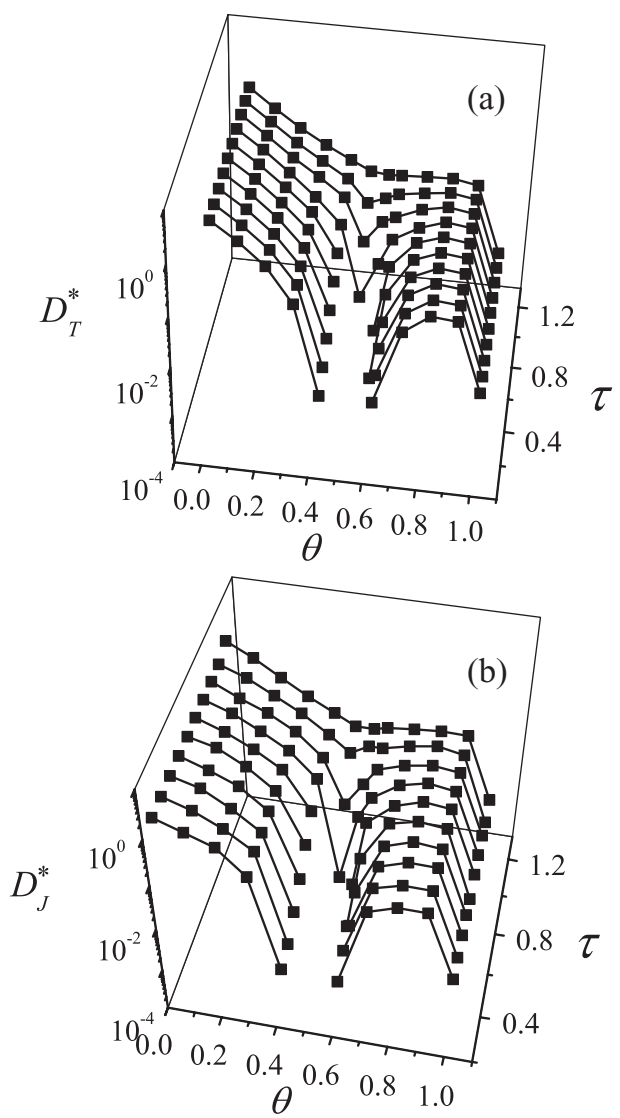

FIG. 9. (a) Normalized tracer diffusion coefficient and (b) normalized jump diffusion coefficient vs $\theta$ and $\tau$ for the interaction kinetics, with $\gamma=-1$ and $C_{i j} \neq 0$. The lower temperature corresponds to $\tau=0.3$ and the temperature step is $\Delta \tau=0.1$.

interactions, the curves have some degree of symmetry around $\theta=0.5$.

The behavior of both diffusion coefficients is very similar to those calculated with a METROPOLIS hopping mechanism, where the hopping probability is proportional to $e^{-\beta\left(E_{f}-E_{i}\right)}$ [here $E_{f}\left(E_{i}\right)$ is the interaction energy in the final (initial) state]. To prove this assumption, we have plotted the jump diffusion coefficient (for $\tau=0.3$ ) using both schemes in Fig. 6. The results are in complete agreement. Note that this METROPOLIS scheme satisfies the PHS.

Next, we discuss the cases in which the PHS is not fulfilled, i.e., $C_{i j}=0$. In Fig. 7, the normalized tracer diffusion coefficient has been represented for $\gamma=1$. As shown in the figure, at low temperature, a minimum appears at $\theta \approx 0.3$ instead of $\theta=0.5$. To explain this, Table I shows the transition rates for different configurations of initial and final states. The first column shows the number of nearest neighbors in the initial and final states for a hopping particle. The second and third columns show its corresponding transition rates. Clearly, the breakdown of PHS leads to an equilibrium condition in which the $c(2 \times 2)$ structure is not favored at half-coverage. Therefore, the diffusion increases with respect to the latter case, as is observed in Fig. 8. This
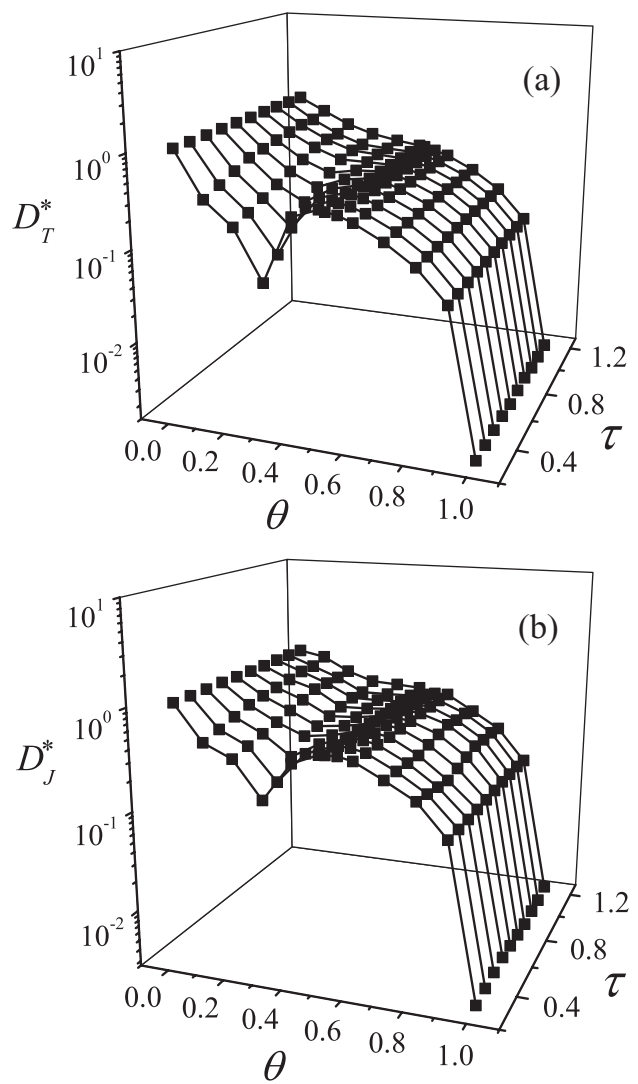

FIG. 10. (a) Normalized tracer diffusion coefficient and (b) normalized jump diffusion coefficient vs $\theta$ and $\tau$ for the interaction kinetics, with $\gamma=1$ and $C_{i j}=0$. The lower temperature corresponds to $\tau=0.3$ and the temperature step is $\Delta \tau=0.1$.

equilibrium condition is obtained starting from any initial configuration.

So far, we have shown the dependence of surface diffusion coefficients with respect to PHS. As we said, there are two coefficients, within the formalism introduced, that are not defined either by the constraints of the PHS or the principle of detailed balance. Considering Eq. (27), different values of the coefficients $C_{11}$ and $C_{22}$ are possible without violating the PHS. Choosing $C_{11}=0$ or $C_{22}=0$, differences in diffusion coefficients in both final-state kinetics as in the interaction kinetics are not observed. However, in the initial-state kinetics there is a strong dependence of the diffusion coefficients with this choice of coefficients. At very low coverages (with repulsive lateral interaction), the involved transition rates do not contain these coefficients, and the diffusion coefficients are independent of the kinetics. This happens at high coverages too. At $\theta=0.5$, the ordered phase is responsible for the diffusion mechanism; this was discussed in the previous paragraphs. This behavior is shown in Fig. 8, where a difference of one order of magnitude in the coefficients can be observed.

An alternative to consider and one that modifies the diffusion coefficient substantially is as follows. For this, the normalized tracer and jump diffusion coefficients [see Figs. 9(a) and 9(b)] for $\gamma=-1$ and $C_{i j} \neq 0$ are analyzed. This value of $\gamma$ belongs to the forbidden region of the phase 


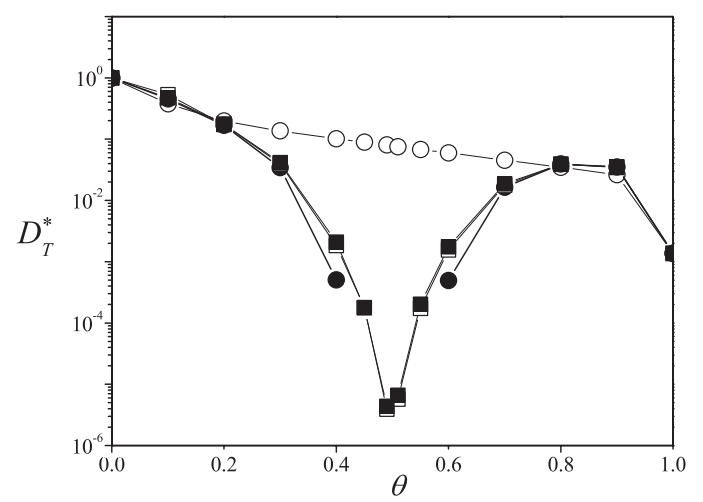

FIG. 11. Influence of the initial configuration in the tracer diffusion coefficient for $C_{i j} \neq 0$ and $\tau=0.3$. Solid squares correspond to $\gamma=1$ and equilibrium initial condition; open squares correspond to $\gamma=1$ and nonequilibrium initial condition. Solid circles correspond to $\gamma=-1$ and equilibrium initial condition; open circles corresponds to $\gamma=-1$ and nonequilibrium initial condition.

diagram. The behavior of both diffusion coefficients is almost the same as for the case described above, except for those values of coverage belonging to the critical region, where no diffusion is observed. The lack of diffusion can be understood by considering the hopping probabilities in both cases, $\gamma=1$ and -1 . Indeed, starting from an equilibrium configuration, the $c(2 \times 2)$ structure is the most probable at half-coverage. In this case, a hopping particle is surrounded by three empty sites in the initial state, while it has three occupied sites in the final state. To compare both cases, for $\tau=0.3$ and $\gamma=1$ the hopping probability corresponding to this configuration is $4.3 \times 10^{-8}$, while for $\gamma=-1$ it is strictly zero. Thus in the first case diffusion exists while in the second case it does not [see Figs. 9 and 10].

To analyze the influence of the initial configuration in the behavior of the diffusion, let us consider the behavior of the tracer diffusion coefficient for both cases $(\gamma=1$ and -1$)$ when the initial configuration is out of equilibrium (random distribution). In Fig. 11, the tracer diffusion coefficient for both cases starting from different initial configurations is shown. In the first case, $\gamma=1$, the initial configuration does not have any influence on the final result, while in the second case, $\gamma=-1$, the diffusion coefficient depends on the initial configuration. As can be observed, the results are completely different.

\section{SUMMARY AND CONCLUSION}

Summarizing, in this work we have analyzed, by means of Monte Carlo simulation, the influence of the PHS on the behavior of the tracer and jump diffusion coefficients for three different hopping mechanisms. We have considered the diffusion of particles in a two-dimensional square lattice with repulsive lateral interactions between nearest neighbors. The hopping mechanisms are determined by the hopping probabilities, which are expressed as a function of the configuration parameters. These parameters depend on the particle interaction in the initial and/or final state.
Depending on the kinetic scheme and the PHS condition, different behaviors of the diffusion coefficient can be obtained. First, the initial-state kinetics, where the PHS is not fulfilled, was analyzed. This is the most common situation in the literature. The results of our study agree with those obtained by other authors.

Thereafter, the effect of the PHS was introduced. In this case, the behavior of both diffusion coefficients is very different, particularly at $\theta>0.5$, where the diffusion coefficient falls several orders of magnitude.

After that, the final-state kinetics without PHS was studied. The result is rarely reported in the literature. Then, the effect of the PHS was also analyzed. For coverages $\theta>0.5$, the behavior of the diffusion coefficient is different. Here the diffusion coefficient rises several orders of magnitude. The curves are almost symmetrical around half-coverage and very similar to the hopping kinetics where initial- and final-state interactions are involved.

Finally, we have studied the interaction kinetics. In this case, the diffusion coefficients depend strongly on both $\gamma$ and the PHS parameters $C_{i j}$. If $\gamma$ belongs to the allowed region of the phase diagram and the PHS is fulfilled, the diffusion coefficients present a strong minimum at $\theta=0.5$, and certain symmetry around half-coverage. This choice of parameters leads to the simple METROPOLIS kinetics, where the hopping probabilities have Arrhenian dependence $(\propto$ $\left.e^{-\beta\left(E_{f}-E_{i}\right)}\right)$. However, if the PHS is not fulfilled, both tracer and jump diffusion coefficients present a minimum at $\theta=0.3$. This equilibrium condition is guaranteed by the principle of detailed balance.

Note that, in the scheme of interaction kinetics, the PHS favors the formation of $c(2 \times 2)$ ordered structure. Furthermore, a modification of the free parameters radically changes the behavior of the coefficients, even if the PHS is fulfilled. As an example, we present the case for the initial state kinetics.

In conclusion, in this work we described different diffusion scenarios depending on the hopping mechanism. We have observed that the principle of detailed balance is a necessary but not sufficient condition for diffusion, therefore the restrictions imposed on the phase diagram $(\gamma, V)$ and the consideration of the PHS are absolutely necessary to obtain physically meaningful diffusion coefficients.

\section{ACKNOWLEDGMENTS}

The authors thank O. Furlong for discussions. This work is partially supported by the CONICET (Argentina).

\section{APPENDIX}

In what follows, the configurations leading to Eqs. (23)(27) corresponding to PHS conditions are shown. In Fig. 12, the solid arrow corresponds to the hopping particle and the dashed arrow corresponds to the hopping hole (vacancy). Equating both rate transitions, the respective equation is obtained. 

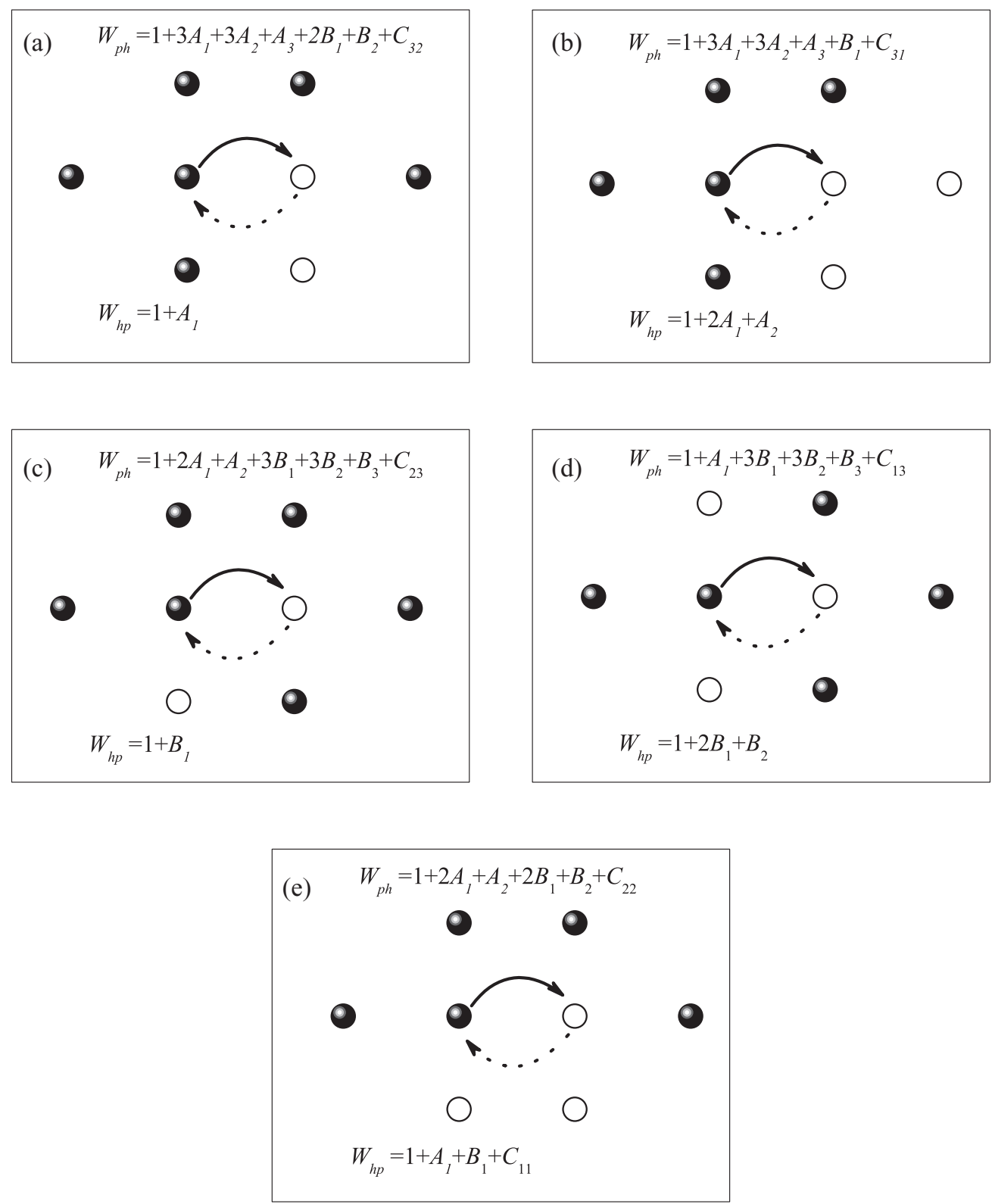

FIG. 12. Schematic representation of the configurations to obtain the PHS conditions. Parts (a)-(e) correspond to Eqs. (23)-(27), respectively.

[1] D. A. Reed and G. Ehrlich, Surf. Sci. 102, 588 (1981); 105, 603 (1981).

[2] R. Gomer, Rep. Prog. Phys. 53, 917 (1990).

[3] J. W. Haus and K. Kehr, Phys. Rep. 150, 263 (1987).

[4] S. Havlin and D. Ben-Avraham, Adv. Phys. 36, 695 (1987).

[5] A. Danani, R. Ferrando, E. Scalas, and M. Torri, Int. J. Mod. Phys. B 11, 2217 (1997).

[6] T. Ala-Nissila, R. Ferrando, and S. C. Ying, Adv. Phys. 51, 949 (2002).

[7] G. Antczak and G. Ehrlich, Surf. Sci. Rep. 62, 39 (2007).

[8] O. M. Braun, T. Dauxois, M. V. Paliy, and M. Peyrard, Phys. Rev. E 55, 3598 (1997).
[9] W. Zwerger, Z. Phys. B 42, 333 (1981); V. Pereyra, A. Milchev, and V. Fleurov, Phys. Rev. E 50, 4636 (1994); G. Zgrablich, V. Pereyra, M. Ponzi, and M. Marchese, AICHE J. 32, 1158 (1986)

[10] A. V. Myshlyavtsev, A. A. Stepanov, C. Uebing, and V. P. Zhdanov, Phys. Rev. B 52, 5977 (1995); C. Uebing and V. P. Zhdanov, Phys. Rev. Lett. 80, 5455 (1998); J. Chem. Phys. 109, 3197 (1998).

[11] C. Uebing, V. Pereyra, and G. Zgrablich, Surf. Sci 366, 185 (1996).

[12] K. Kawazaki, Phys. Rev. 145, 224 (1966).

[13] Z. Chvoj, J. Phys.: Condens. Matter 12, 2135 (2000). 
[14] A. A. Chumak and A. A. Tarasenko, Surf. Sci. 91, 694 (1980).

[15] S. Glasstone, K. J. Laidler, and H. Eyring, The Theory of Rate Processes (McGraw-Hill, New York, 1941).

[16] V. P. Zhdanov, Elementary Physicochemical Processes on Solid Surfaces (Plenum, New York, 1991).

[17] V. P. Zhdanov, Surf. Sci. 149, L13 (1985).

[18] V. P. Zhdanov, Surf. Sci. 111, 63 (1981); 111, L662 (1981); 123, 106 (1982); 133, 469 (1983); 157, L384 (1985); 165, L31 (1986); 171, L461 (1986); 209, 523 (1989); Surf. Sci. Rep. 12, 185 (1991)

[19] B. Li, C.-S. Zhang, V. P. Zhdanov, and P. R. Norton, Surf. Sci. 322, 373 (1995).

[20] V. P. Zhdanov and B. Kasemo, Surf. Sci. 412, 527 (1998).

[21] S. J. Manzi, R. E. Belardinelli, G. Costanza, and V. D. Pereyra, Phys. Rev. E. 79, 021103 (2009).

[22] S. J. Manzi, V. J. Huespe, R. E. Belardinelli, and V. D. Pereyra, Phys. Rev. E. 80, 051112 (2009).

[23] Z. W. Gortel and M. A. Zaluska-Kotur, Phys. Rev. B 70, 125431 (2004); L. Badowski, M. A. Zaluska-Kotur, and Z. W. Gortel, ibid. 72, 245413 (2005); 72, 235425 (2005); 74, 045405 (2006); M. Yakes, M. Hupalo, M. A. Zaluska-Kotur, Z. W. Gortel, and M. C. Tringides, Phys. Rev. Lett. 98, 135504 (2007).

[24] P. Argyrakis, A. A. Chumak, and M. Maragakis, Phys. Rev. B 71 , 224304 (2005); 76, 054209 (2007); P. Argyrakis, M. Maragakis, O. Chumak, and A. Zhugayevych, ibid. 74, 035418 (2006); P. Argyrakis and A. A. Chumak, ibid. 66, 054303 (2002).

[25] S. H. Payne and H. J. Kreuzer, Phys. Rev. B 75, 115403 (2007).

[26] S. H. Payne and H. J. Kreuzer, Phys. Rev. B 77, 121403(R) (2008).
[27] S. H. Payne and H. J. Kreuzer, J. Phys: Condens. Matter 21, 134013 (2009).

[28] S. J. Manzi, G. A. Ranzuglia, and V. D. Pereyra, Phys. Rev. E 80, 062104 (2009).

[29] J. J. Torrez Herrera, G. A. Ranzuglia, S. J. Manzi, and V. D. Pereyra, Phys. Rev. E 84, 051102 (2011).

[30] N. B. Wilding, Z. Phys. B 93, 119 (1993).

[31] D. P. Landau and K. Binder, Phys. Rev. B 31, 5946 (1985).

[32] H. J. Kreuzer and S. H. Payne, in Computational Methods in Colloid and Interface Science, edited by M. Borowko (Dekker, New York, 1999), pp. 439-479.

[33] H. J. Kreuzer, Langmuir 8, 774 (1992).

[34] C. Uebing and R. Gomer, J. Chem. Phys. 95, 7626 (1991); 95, 7636 (1991); 95, 7641 (1991); 95, 7648 (1991); C. Uebing, V. Pereyra, and G. Zgrablich, Surf. Sci. 366, 185 (1996).

[35] F. M. Bulnes, V. D. Pereyra, and J. L. Riccardo, Phys. Rev. E 58, 86 (1998).

[36] A. J. Ramirez-Pastor, T. P. Eggarter, V. D. Pereyra, and J. L. Riccardo, Phys. Rev. B 59, 11027 (1999).

[37] T. E. Harris, J. Appl. Prob. 2, 323 (1965).

[38] K. Binder and D. P. Landau, Surf. Sci. 108, 503 (1981).

[39] W. Kinzel, W. Selke, and K. Binder, Surf. Sci. 121, 13 (1982).

[40] A. Patrykiejew, S. Sokolowski, and K. Binder, Surf. Sci. Rep. 38, 207 (2000).

[41] Equations (16)-(27) form a linearly compatible undetermined system. After using simple algebraic calculations, one can transform the coefficient matrix to an invertible $10 \times 10$ matrix. Therefore, two of the coefficients can be determined arbitrarily. 